\title{
ON THE CORRELATION OF THE FIR AND RADIO RADIATION OF SPIRAL GALAXIES \\ H.M.Tovmassian
}

INAOE, AP 51 y 216, 72000, Puebla, Pue, Mexico

The comparison of the Byurakan classification of central parts of galaxies with the results of their radio observations showed that there is a definite correlation: the radio emission is more of ten observed in those galaxies which were suggested to have active nuclei.

In this talk the data on the IRAS bright galaxy sample (Soifer et al. 1987) are compared with the Byurakan classes of spiral galaxies. Total number of spiral galaxies in the Byurakan list brighter than $13 .{ }^{m} 5$ is 525 and that of in the IRAS list common to the Byurakan catalogue is 128. The ratios of relative numbers of galaxies of the IRAS galaxies of different Byurakan types to that of galaxies from Byurakan list, normalized to unity for galaxies of type 3 , are shown below:

$$
\begin{array}{lcccccr}
\text { Byurakan type } & 3 & 1 & 4 & 2 & 5 & 2 \mathrm{~s} \\
\text { IRAS/Byurakan } & 1.00 & 1.38 & 2.89 & 3.33 & 3.80 & 6.67
\end{array}
$$

Thus galaxies of types $4,2,5$ and $2 \mathrm{~s}$, which have optical manifestations of nuclear activity and more of ten have radio emission, appreciably more of ten have also FIR radiation. The FIR luminosity itself is depending on the degree of activity expressed by their Byurakan types. The mean values of the $\mathrm{m}_{\mathrm{v}}-\mathrm{m}_{60}$ colors of galaxies are changing from -1.32 for type 3 galaxies to -0.42 for $2 \mathrm{~s}$ galaxies when moving from type 1 towards types 3, 4, 5, 2 and $2 \mathrm{~s}$. The same trend is seen in in the case of $m_{v}-m_{100}$ colors: from -0.33 to 0.35 (The FIR stellar magnitudes are in arbitrary units).

Thus the FIR emission of spiral galaxies is definitely correlated with their Byurakan types. And since the radio emission of spiral galaxies is also correlated with their Byurakan types (Tovmassian $1982)$ it is natural to expect that the $F I R$ and radio radiations should also be correlated. It shows that the Byurakan classification based on the optical appearances of the central parts of galaxies revealed the galaxies with an active nuclei and star burst processes.

The author is indebted to CONACYT for support of this work.

\section{REFERENCES}

Sofier B.T., Sanders D.B., Madore B.F., Neugebauer G., Danielson G.E., Elias J.H., Lonsdale C. \& Rice W.L. 1987. ApJ, 320, 238.

Tovmassian H.M. 1982. Astrof isika, 18, 25. 